\title{
ANKLE
}

\section{Anatomy of the ankle ligaments: a pictorial essay}

\author{
Pau Golanó · Jordi Vega • Peter A. J. de Leeuw • \\ Francesc Malagelada - M. Cristina Manzanares • \\ Víctor Götzens $\cdot$ C. Niek van Dijk
}

Received: 13 February 2010/ Accepted: 16 February 2010/Published online: 23 March 2010

(C) The Author(s) 2010. This article is published with open access at Springerlink.com

\begin{abstract}
Understanding the anatomy of the ankle ligaments is important for correct diagnosis and treatment. Ankle ligament injury is the most frequent cause of acute ankle pain. Chronic ankle pain often finds its cause in laxity of one of the ankle ligaments. In this pictorial essay, the ligaments around the ankle are grouped, depending on their anatomic orientation, and each of the ankle ligaments is discussed in detail.
\end{abstract}

Keywords Ankle anatomy - Lateral collateral ligament . Medial collateral ligament - Ankle impingement . Ankle sprain

\section{P. Golanó ( $₫)$}

Laboratory of Arthroscopic and Surgical Anatomy,

Department of Pathology and Experimental Therapeutics

(Human Anatomy Unit), University of Barcelona,

c/Feixa Llarga s/n (Campus Bellvitge),

08907 L'Hospitalet de Llobregat, Barcelona, Spain

e-mail: pgolano@ub.edu

M. C. Manzanares · V. Götzens

Department of Pathology and Experimental Therapeutics

(Human Anatomy Unit), University of Barcelona,

c/Feixa Llarga s/n (Campus Bellvitge),

08907 L'Hospitalet de Llobregat, Barcelona, Spain

e-mail: mcmanzanares@ub.edu

V. Götzens

e-mail: vgotzens@ub.edu

J. Vega

Department of Orthopedic and Trauma Surgery,

Hospital Asepeyo San Cugat,

Avenida Alcalde Barnils 54-56,

08174 San Cugat del Vallès, Barcelona, Spain

e-mail: jordivega@hotmail.com

\section{Introduction}

Despite the fact that the ankle ligaments are prone to injury during the fast majority of sports, literature focusing on the ankle ligaments is rare. Proper anatomic knowledge of the different ligaments is important for a correct diagnosis and subsequent treatment.

The most common mechanism of injury to the ankle ligaments is inversion of the foot [4, 33]. With this mechanism of injury, the anterior talofibular ligament is the first or only ligament to sustain injury [43]. A total rupture involves the calcaneofibular ligament and the posterior talofibular ligaments as well [9]. An eversion injury will

\author{
P. A. J. de Leeuw - C. N. van Dijk \\ Department of Orthopaedic Surgery, \\ Academic Medical Center, University of Amsterdam, \\ PO Box 22700, 1100 DE Amsterdam, The Netherlands \\ e-mail: p.a.deleeuw@amc.uva.nl \\ C. N. van Dijk \\ e-mail: c.n.vandijk@amc.uva.nl \\ F. Malagelada \\ Department of Orthopedic and Trauma Surgery, \\ Hospital de Mataró, Carretera de Cirera s/n, \\ 08304 Mataró, Barcelona, Spain \\ e-mail: fmalagelada@gmail.com
}


cause damage to the deltoid ligaments [3], while a hyperdorsiflexion trauma might cause an injury to the syndesmotic ligaments [11].

The ligaments around the ankle can be divided, depending on their anatomic position, into three groups: the lateral ligaments, the deltoid ligament on the medial side, and the ligaments of the tibiofibular syndesmosis that join the distal epiphyses of the bones of the leg (tibia and fibula).

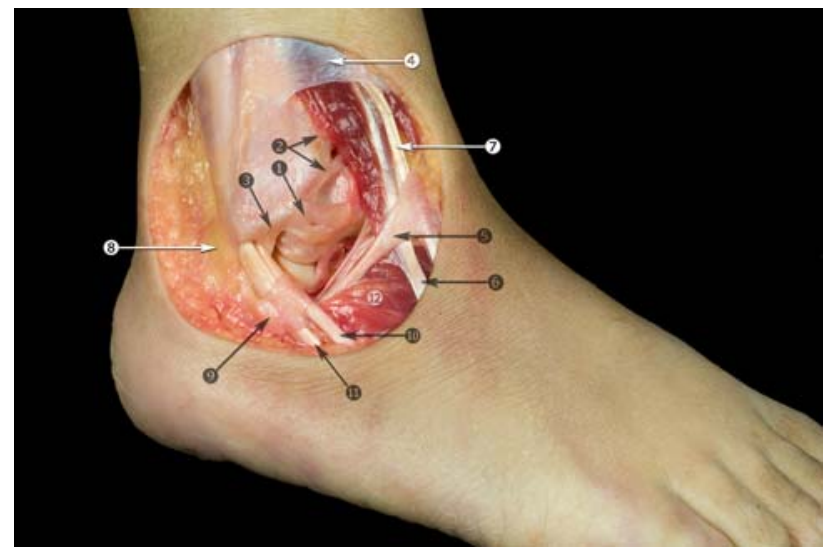

Fig. 1 Anterolateral view of the ankle. Anatomic dissection. 1 Anterior talofibular ligament; 2 anterior tibiofibular ligament; 3 fibular insertion of the calcaneofibular ligament; 4 superior extensor retinaculum; 5 inferior extensor retinaculum; 6 peroneus tertius tendon; 7 extensor digitorum longus tendons; 8 superior peroneal retinaculum; 9 inferior peroneal retinaculum; 10 peroneus brevis tendon; 11 peroneus longus tendon; 12 extensor digitorum brevis muscle

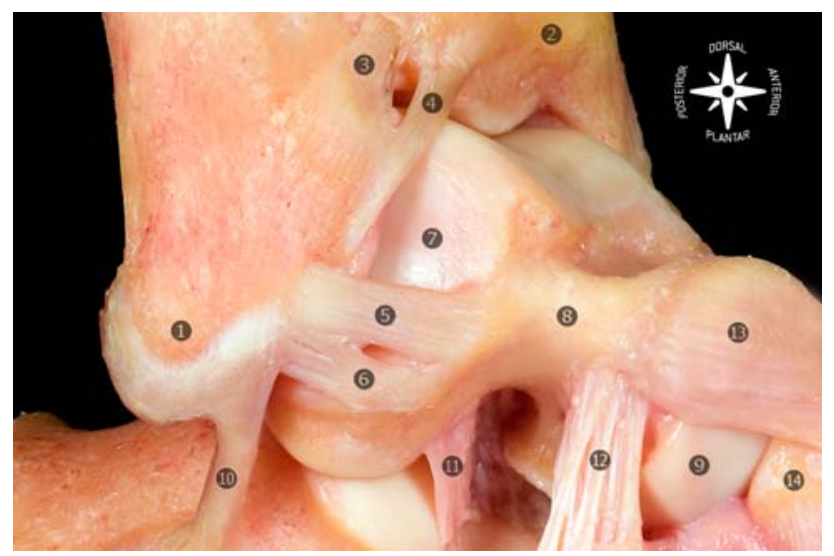

Fig. 2 Osteoarticular anatomic dissection of the lateral ligaments of the foot and ankle joint. The anterior talofibular ligament is typically composed of two separate bands. 1 Tip of the lateral malleolus; 2 tibia; 3 anterior tibiofibular ligament; 4 distal fascicle of the anterior tibiofibular ligament; 5 superior band of the anterior talofibular ligament; 6 inferior band of the anterior talofibular ligament; 7 lateral articular surface of the talus; 8 neck of the talus; 9 head of the talus; 10 calcaneofibular ligament; 11 talocalcaneal interosseous ligament; 12 cervical ligament; 13 talonavicular ligament; 14 navicular
In this review article, these three groups of ligaments are described separately, and in each section, the specific ligaments are described in detail.

\section{The lateral and medial collateral ligaments}

The lateral collateral ligament complex (LCL) consists of the anterior talofibular, the calcaneofibular, and the posterior talofibular ligaments. The medial collateral ligaments (MCL), also known as the deltoid ligament, are a multifascicular group of ligaments and can roughly be divided into a superficial and deep group of fibers $[8,24,28,36]$.

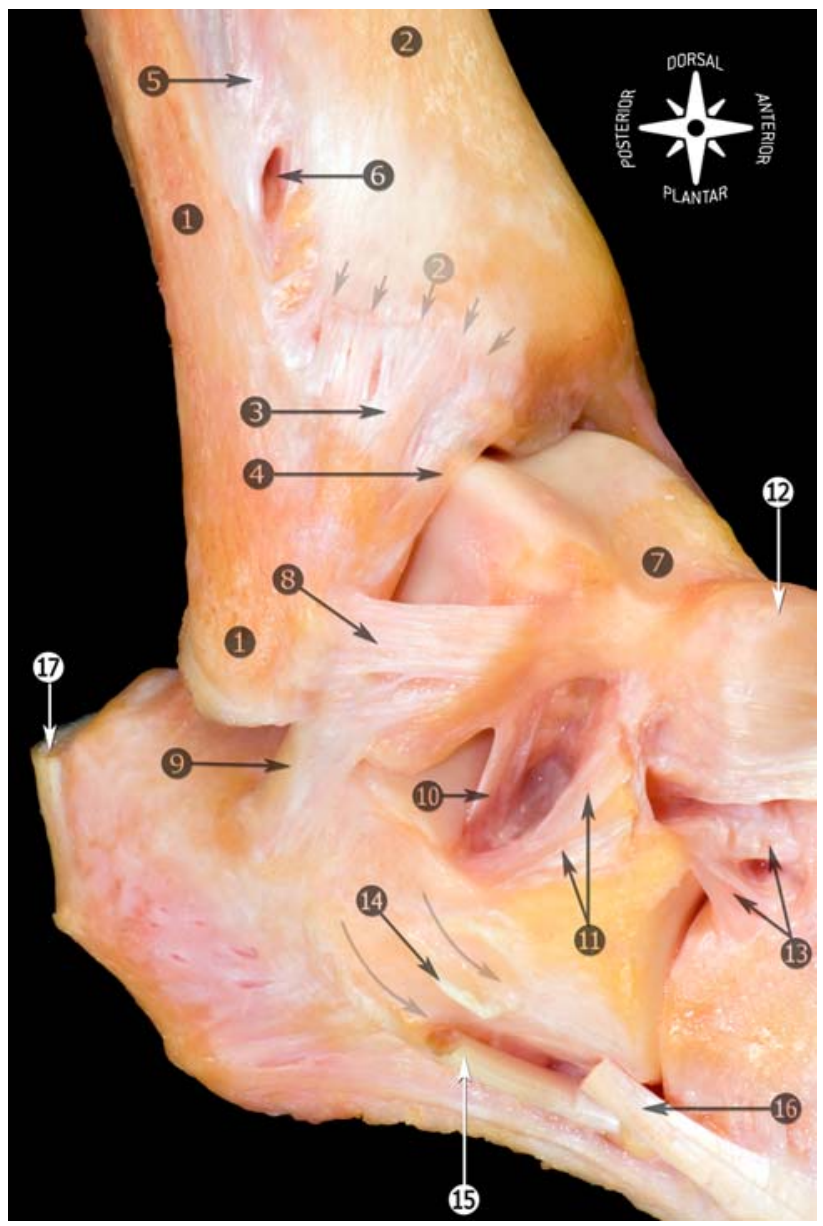

Fig. 3 Anatomic dissection of the lateral region of the foot and ankle showing the morphology and relationship of the anterior talofibular with the calcaneofibular ligaments. 1 Fibula and tip of the fibula; 2 tibia (anterior tubercle with arrows); 3 anterior tibiofibular ligament; 4 distal fascicle of the tibiofibular ligament; 5 interosseous membrane; 6 foramen for the perforating branch of the peroneal artery; 7 talus; 8 anterior talofibular ligament; 9 calcaneofibular ligament; 10 talocalcaneal interosseous ligament; 11 inferior extensor retinaculum (cut); 12 talonavicular ligament; 13 bifurcate ligament; 14 peroneal tubercle (arrows showing the peroneal tendons sulcus); 15 peroneus longus tendon; 16 peroneus brevis tendon; 17 calcaneal tendon 


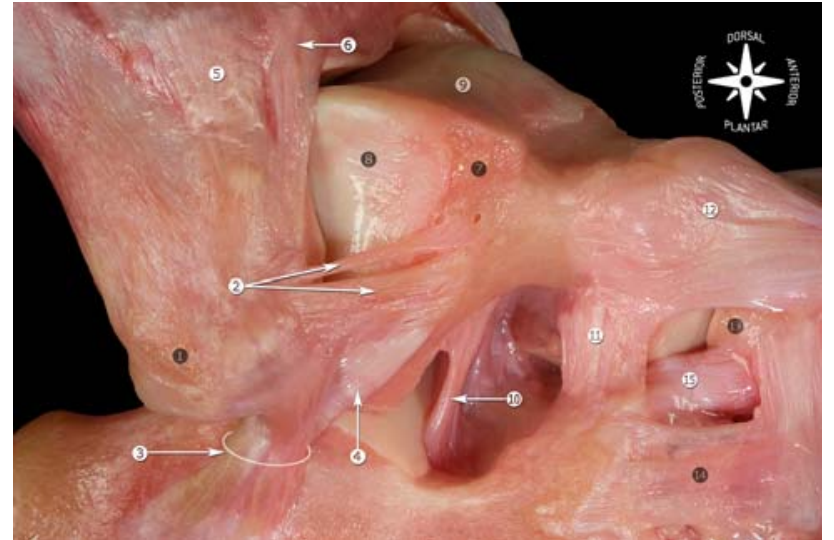

Fig. 4 Anatomic dissection of the lateral ankle ligaments showing the relationship of the calcaneofibular and lateral talocalcaneal ligaments with the morphology of the anterior talofibular ligament. Some authors describe a third band of the anterior talofibular ligament. We have never found this third band in our dissections. In the presented dissection, the superior band of the anterior talofibular ligament is smaller than usually. 1 Tip of the fibula; 2 superior and inferior bands of the anterior talofibular ligament; 3 calcaneofibular ligament; 4 lateral talocalcaneal ligament; 5 anterior tibiofibular ligament; 6 distal fascicle of the anterior tibiofibular ligament; 7 triangular region of the talus; 8 lateral articular surface of the talus; 9 dorsal articular surface of the talus; 10 talocalcaneal interosseous ligament; 11 cervical ligament; 12 talonavicular ligament; 13 navicular; 14 lateral calcaneocuboid ligament; 15 bifurcate ligament (calcaneonavicular fascicle)

\section{Lateral collateral ligaments}

\section{Anterior talofibular ligament}

The anterior talofibular ligament is the most frequently injured ligament of the ankle and is the most frequently observed injury in the emergency room [7] (Fig. 1). This ligament plays an important role in limiting anterior displacement of the talus and plantar flexion of the ankle [40].

This ligament is closely related to the ankle joint capsule and is typically composed of two separate bands [23] (Fig. 2). The bands are separated by vascular branches from the perforating peroneal artery and its anastomosis with the lateral malleolar artery. In literature, numerous anatomic descriptions have been given, varying from a single up to three bands [9, 10, 12, 23, 24]; however, in our observation during ankle dissections, this ligament most commonly compromises a double-banded morphology, similar to the description by Sarrafian [36].

The anterior talofibular ligament originates at the anterior margin of the lateral malleolus. The center is on average $10 \mathrm{~mm}$ proximal to the tip of the fibula as measured along the axis of the fibula [10]. The overall width $(6-10 \mathrm{~mm})$ of the anterior tibiotalar ligament does not appear to vary greatly irrespective of the number of bands present, suggesting that the variations observed do not modify the ligament's function [40] (Figs. 3, 4).

From its origin, it runs anteromedially to the insertion on the talar body immediately anterior to the joint surface occupied by the lateral malleolus. The ligament is virtually horizontal to the ankle in the neutral position but inclines upward in dorsiflexion and downward in plantar flexion. It is only in the latter position that the ligament comes under strain and is vulnerable to injury, particularly, when the foot is inverted [9].

In plantar flexion, the inferior band of the ligament remains relaxed while the upper band becomes taut. In dorsiflexion, the upper band remains relaxed, and the inferior band becomes tight.
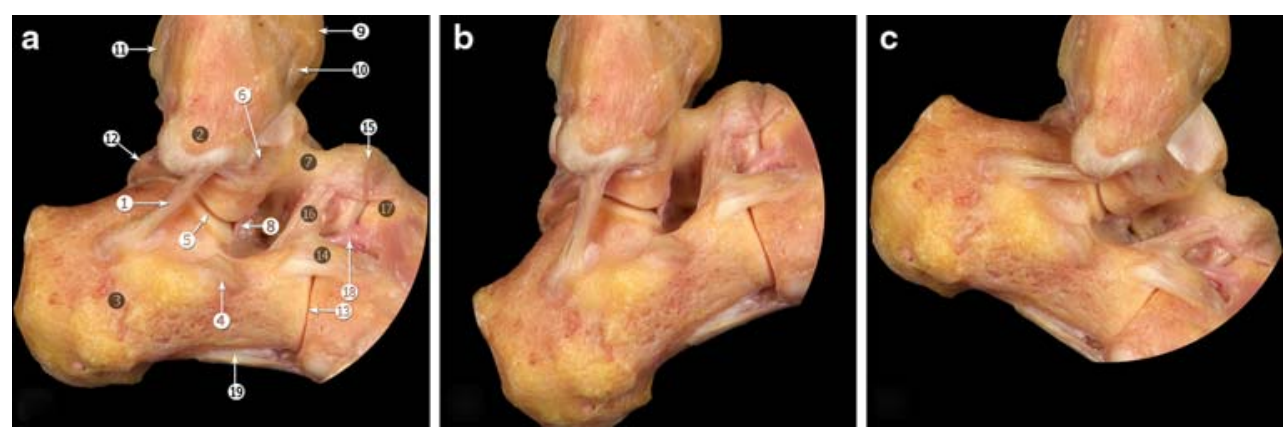

Fig. 5 Osteoarticular dissection of the calcaneofibular ligament during ankle movements. a Neutral position. b Dorsal flexion. c Plantar flexion. Calcaneofibular ligament becomes horizontal during plantar flexion and vertical in dorsal flexion, remaining tensed throughout the entire arc of motion of the ankle. 1 Calcaneofibular ligament; 2 tip of the fibula; 3 calcaneus; 4 peroneal tubercle; 5 subtalar joint; 6 anterior talofibular ligament; 7 neck of the talus;
8 talocalcaneal interosseous ligament; 9 anterior tubercle of the tibia; 10 anterior tibiotalar ligament; 11 posterior tubercle of the tibia; 12 lateral talar process; 13 calcaneocuboid joint; 14 lateral calcaneocuboid ligament; 15 talonavicular ligament; 16 cervical ligament; 17 navicular; 18 bifurcate ligament (calcaneonavicular fascicle); 19 long plantar ligament 


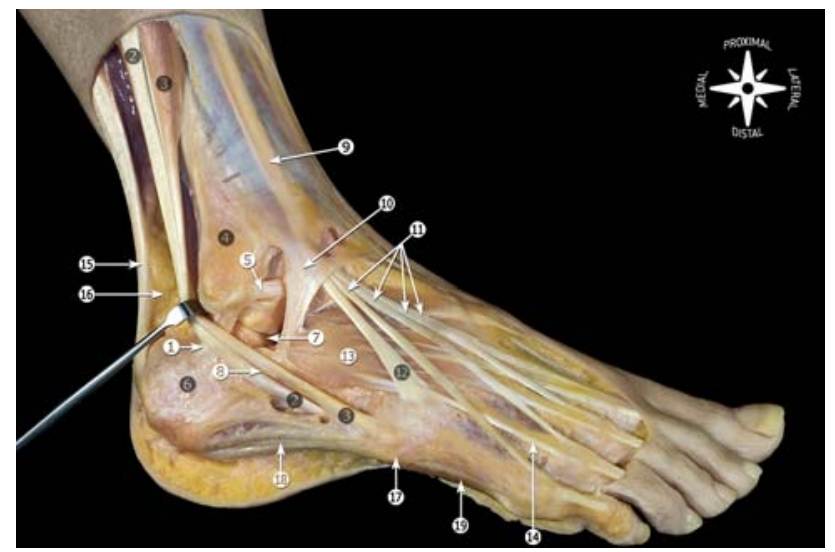

Fig. 6 Anatomic dissection showing the relationship of the calcaneofibular ligament with peroneal tendons. 1 Calcaneofibular ligament; 2 peroneus longus tendon; 3 peroneus brevis tendon; 4 fibula; 5 talofibular ligament; 6 calcaneus; 7 subtalar joint; 8 septum in the peroneal tubercle; 9 superior extensor retinaculum; 10 inferior extensor retinaculum; 11 extensor digitorum longus tendons; 12 peroneus tertius tendon; 13 extensor digitorum brevis; 14 extensor digitorum brevis tendon; 15 calcaneal tendon; 16 Kager's fat pad; 17 tuberosity of the fifth metatarsal bone; 18 lateral plantar fascia; 19 abductor digiti minimi

\section{Calcaneofibular ligament}

The calcaneofibular ligament originates from the anterior part of the lateral malleolus. It is anatomically positioned just below the lower band of the anterior talofibular ligament. Frequently, fibers connecting these ligaments can be observed (Fig. 3). In the neutral ankle position, the ligament runs obliquely downwards and backwards to attach to the posterior region of the lateral calcaneal surface (Fig. 5). This ligament is superficially crossed by the peroneal tendons and sheaths, which can leave a concavity over the ligament; only about $1 \mathrm{~cm}$ of the ligament is uncovered (Fig. 6).

The anatomic variants of the calcaneofibular ligament and their relationship with the lateral talocalcaneal ligament have been the subject of study [39]. In 35\% of the cases, the calcaneofibular ligament is reinforced by the lateral talocalcaneal ligament, attached by the former but diverging proximally or distally. In $23 \%$ of the cases, a lateral talocalcaneal ligament exists anteriorly and independent of the calcaneofibular ligament. In $42 \%$ of the cases, the lateral talocalcaneal is absent and is replaced by an anterior talocalcaneal ligament. In these cases, the calcaneofibular ligament acquires more functional significance in providing stability to the subtalar joint [39] (Fig. 7).

In cross-section, the ligament is rounded and has a diameter of $6-8 \mathrm{~mm}$, and its length is about $20 \mathrm{~mm}$. This ligament is separate from the ankle joint capsule, but it is intimately associated with the posteromedial part of the

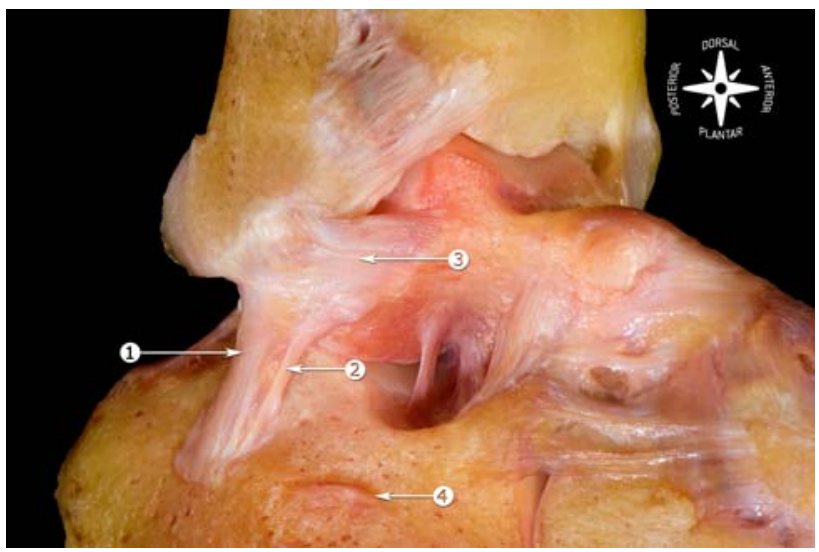

Fig. 7 Osteoarticular dissection. Relationship of the calcaneofibular ligament with the lateral talocalcaneal ligament. 1 Calcaneofibular ligament; 2 lateral talocalcaneal ligament; 3 anterior talofibular ligament; 4 peroneal tubercle

peroneal tendons sheath, covering almost the entire ligament [36].

The calcaneofibular ligament is the only ligament bridging both the talocrural joint and subtalar joint. Insertion of this ligament and its axis of rotation point allow flexion and extension movements of the talocrural joint. Depending on its bi-articular characteristic, this ligament also permits subtalar movement.

Broström found that combined ruptures of the anterior talofibular and the calcaneofibular ligaments occurred in $20 \%$ of cases and that isolated rupture of the calcaneofibular ligament was very rare [9]. The posterior talofibular ligament is usually not injured unless there is a frank dislocation of the ankle.

Variants in its orientation of the calcaneofibular ligament were studied by Ruth [35]. The calcaneofibular ligament becomes horizontal during extension and vertical in flexion, remaining tense throughout its entire arc of motion (Fig. 5). A valgus or varus position of the talus considerably changes the angle formed by the ligament and the longitudinal axis of the fibula. The ligament is relaxed in the valgus position and tense in the varus position. This explains the potential for injury even without dorsiflexionplantar flexion movement in the ankle.

\section{Posterior talofibular ligament}

The posterior talofibular ligament originates from the malleolar fossa, located on the medial surface of the lateral malleolus, coursing almost horizontally to insert in the posterolateral talus. In plantar flexion and in the neutral ankle position, the ligament is relaxed, while in dorsiflexion, the ligament is tensed. Due to the multifascicular aspect of this ligament, it inserts not just in a specific area. Fibers insert in the posterior surface of the talus, in the 


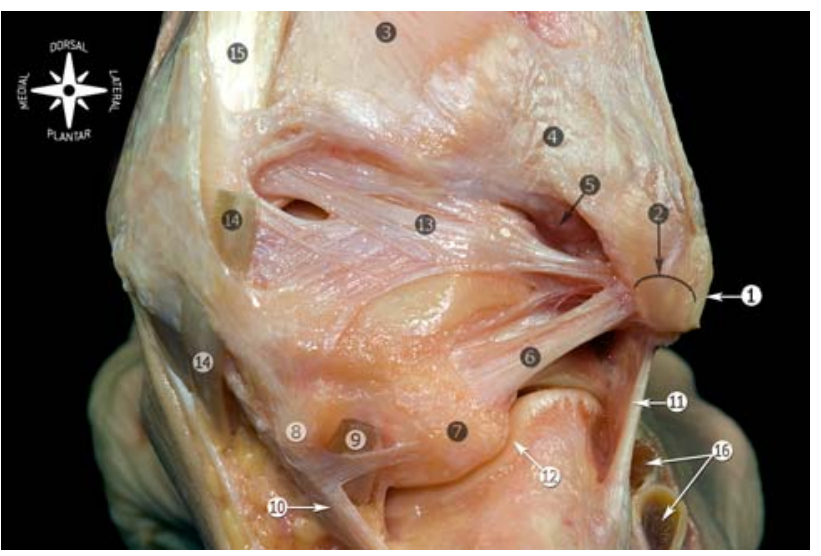

Fig. 8 Posterior view of the anatomic dissection of the ankle ligaments. 1 Tip of the fibula; 2 peroneal groove of the fibula; 3 tibia; 4 superficial component of the posterior tibiofibular ligament; 5 deep component of the posterior tibiofibular ligament or transverse ligament; 6 posterior calcaneofibular ligament; 7 lateral talar process; 8 medial talar process; 9 tunnel for flexor hallucis longus tendon; 10 flexor hallucis longus retinaculum; 11 calcaneofibular ligament; 12 subtalar joint; 13 posterior intermalleolar ligament; 14 flexor digitorum longus tendon (cut); 15 tibialis posterior tendon; 16 peroneal tendons

lateral talar process or os trigonum, if present. Some fibers can contribute in forming the tunnel for the flexor hallucis longus tendon (Fig. 8).

Moreover, a group of fibers fuse with the posterior intermalleolar ligament [29]. The posterior intermalleolar ligament has been the subject of recent studies because of its involvement in the posterior soft tissue impingement syndrome of the ankle [17, 27]. Its prevalence of occurrence both in radiological and in anatomic studies vary widely, ranging from $19 \%$ up to $100 \%$ [24, 27, 30]. In our dissections, the intermalleolar ligament is a consistent finding [16].

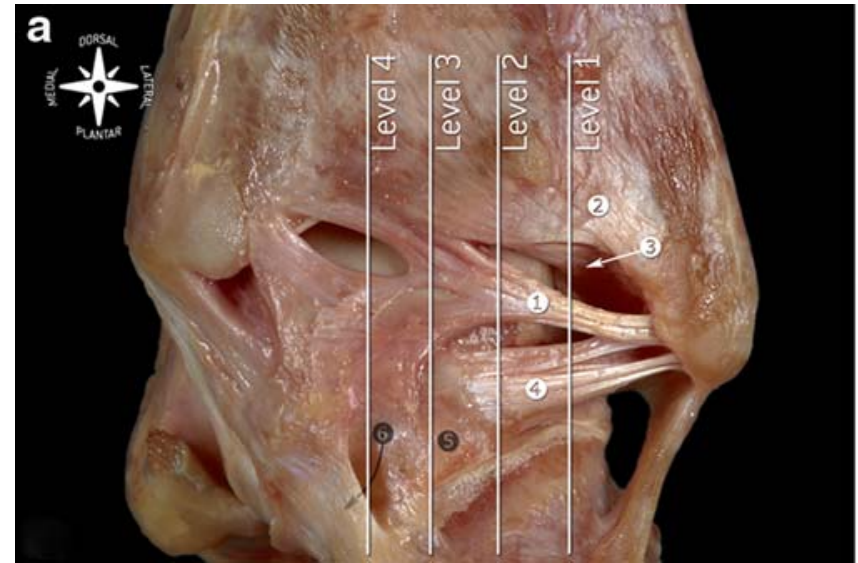

Fig. 9 a Posterior view of the ankle ligaments showing the relationships of the posterior intermalleolar ligament, posterior talofibular ligament and transverse ligament. b T1-weighted spin-echo MR imaging showing the correlation between MRI and the saggital cuts in
In the recent study of Oh et al. [27] on the morphology of the posterior intermalleolar ligament and its correlation with MR images, the posterior intermalleolar ligament was observed in $81.8 \%$ of the specimens (63 of 77 specimens). In MRI study, the intermalleolar ligament was identified in all ankles (26 specimens) (Figs. 9, 10).

These differences can probably be explained by its limited size and therefore difficult assessment during an ankle dissection, although $\mathrm{Oh}$ et al. [27] propose the number of specimens used or interracial variations. In addition, the ligament may be divided into two or three different bands (20\% [34]-100\% [27]).

In the posterior view, the posterior intermalleolar ligament is situated between the transverse ligament and the posterior talofibular ligament and runs obliquely from lateral to medial and from downwards to upwards. The shape of the posterior intermalleolar ligament is variable. These variations depend on its medial arising sites, the number of composing fiber bundles, and the degree of the bundle compactness. The medial arising sites of the ligament included the lateral border of the medial malleolar sulcus, the medial border of the medial malleolar sulcus through the septum between the flexor digitorum longus and posterior tibial tendons, the posterior distal margin of the tibia, and the posterior process of the talus through the joint capsule [27] (Fig. 10). The posterior intermalleolar ligament tenses during dorsiflexion and relaxes during plantar flexion, and therefore, trauma that causes forced dorsiflexion of the ankle can be assumed to produce either injury or rupture of this ligament, or osteochondral avulsion. Plantar flexion would cause it to relax and become susceptible to trapping between the tibia and the talus, leading to impingement (Fig. 11).

Since the introduction of the posterior ankle arthroscopy by van Dijk et al. [44, 45] in 2000, the posterior ankle

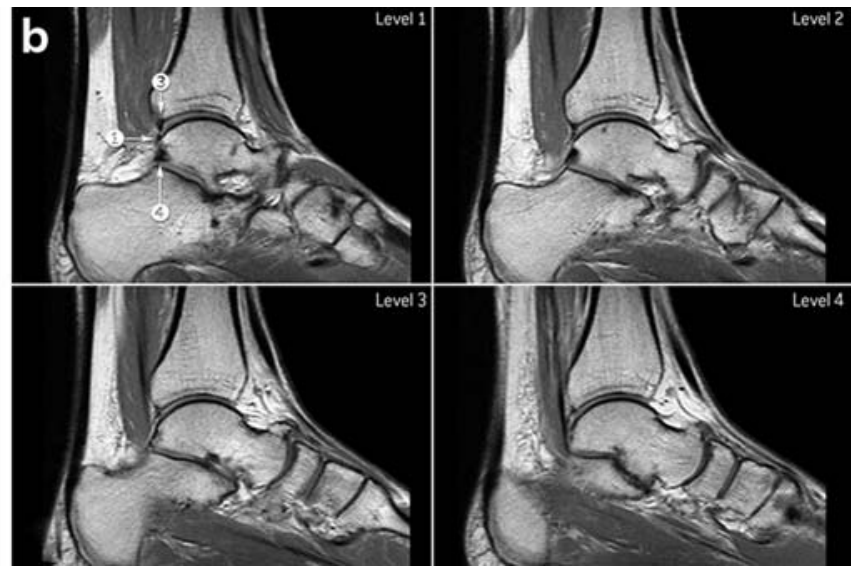

a. 1 Posterior intermalleolar ligament; 2 superficial component of the tibiofibular ligament; 3 deep component of the tibiofibular ligament or transverse ligament; 4 posterior talofibular ligament; 5 lateral talar process; 6 tunnel for flexor hallucis longus tendon 


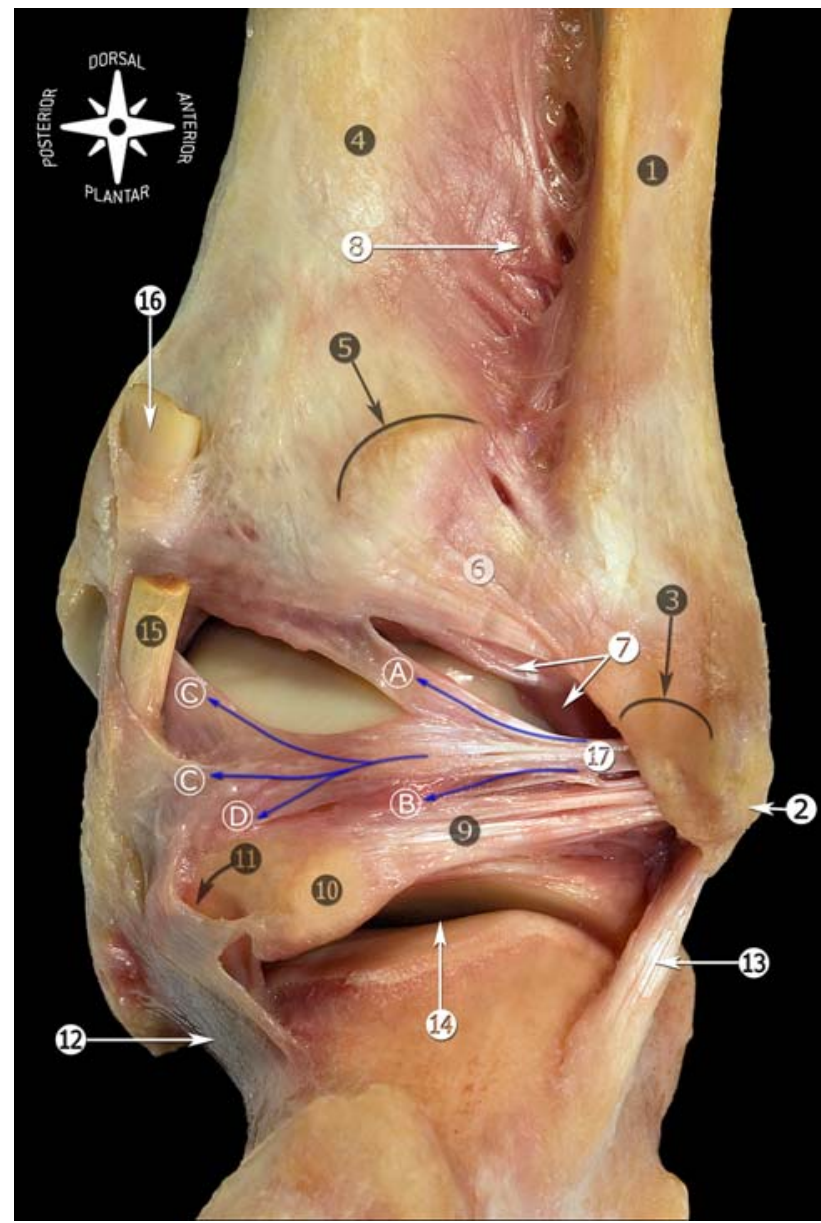

Fig. 10 Posterior view of the anatomic dissection of the ankle ligaments showing the posterior intermalleolar ligament with its relation to the surrounding anatomy. 1 Fibula; 2 tip of the fibula; 3 peroneal groove of the fibula; 4 tibia; 5 posterior tubercle of the tibia; 6 superficial component of the posterior tibiofibular ligament; 7 deep component of the posterior tibiofibular ligament or transverse ligament; 8 interosseous membrane; 9 posterior talofibular ligament; 10 lateral talar process; 11 tunnel for flexor hallucis longus tendon; 12 flexor hallucis longus retinaculum; 13 calcaneofibular ligament; 14 subtalar joint; 15 flexor digitorum longus tendon (cut); 16 tibialis posterior tendon (cut); 17 posterior intermalleolar ligament: $A$ Tibial insertion (tibial slip in arthroscopic view). $B$ Talar insertion (lateral talar process). $C$ Tibial malleolar insertion through the septum between the flexor digitorum longus and posterior tibial tendons. $D$ Talar insertion (medial talar process) through the joint capsule

ligaments can clearly be visualized and treated in case of pathology.

\section{Medial collateral ligament}

The anatomical descriptions of the MCL vary widely in the literature; however, in general most agree that it is composed of two layers; the superficial and deep [8, 24, 28, 36].
Similar to the posterior talofibular ligament, the MCL is a multifascicular ligament, originating from the medial malleolus to insert in the talus, calcaneus, and navicular bone.

The tendon sheath of the posterior tibial muscle covers the posterior and middle part of the deltoid ligament in much the same way as the peroneal tendon sheath is associated with the calcaneofibular ligament on the lateral side.

The most commonly accepted description of the MCL is the one originally proposed by Milner and Soames [24]. Six bands or components have been described for the MCL: three of them are always present (the tibiospring ligament, tibionavicular ligament, and deep posterior tibiotalar ligament), whereas the presence of the other three may vary (superficial posterior tibiotalar ligament, tibiocalcaneal ligament, and deep anterior tibiotalar ligament) $[8,24]$ (Table 1; Figs. 12, 13). Most of the MCL is covered by tendons as it extends down the leg to the bony insertions in the foot (Fig. 14).

Although the description proposed by Milner and Soames has been accepted [24], the anatomy of this ligament and its components is still confusing. During our dissections, we found it rather difficult to determine each individual band, since most are continuous to one another, and therefore pointing out individual bands is artificial.

\section{Ligaments that join the distal epiphyses of the tibia and fibula}

The talocrural joint consists of a fork-shaped dome formed by the distal tibia and fibula and the talar trochlea enclosed by this mortise. Cartilagenous areas of the ankle joint are not congruent in their surface outlines. In the frontal plane, the talar dome has a slightly concave profile. The planes of the tibial and fibular facets are not parallel. The trochlea is wider anteriorly than posteriorly, and the cartilage covered surfaces have slightly curved sides. The fibular facet has a convex contour, whereas the tibial facet is concave [13].

It is a syndesmotic articulation that allows the tibiafibula as a whole to adapt to the varying width of the upper articular surface of the talus by slight ascending and medial rotation movements of the fibula during extreme dorsiflexion (maximum width) and by inverse movements during plantar flexion (minimum width) [21].

The syndesmotic ligament complex ensures the stability between the distal tibia and the fibula and resists the axial, rotational, and translational forces that attempt to separate the tibia and fibula. The three ligaments responsible are the anterior or anteroinferior tibiofibular ligament, the posterior or posteroinferior tibiofibular ligament, and the 

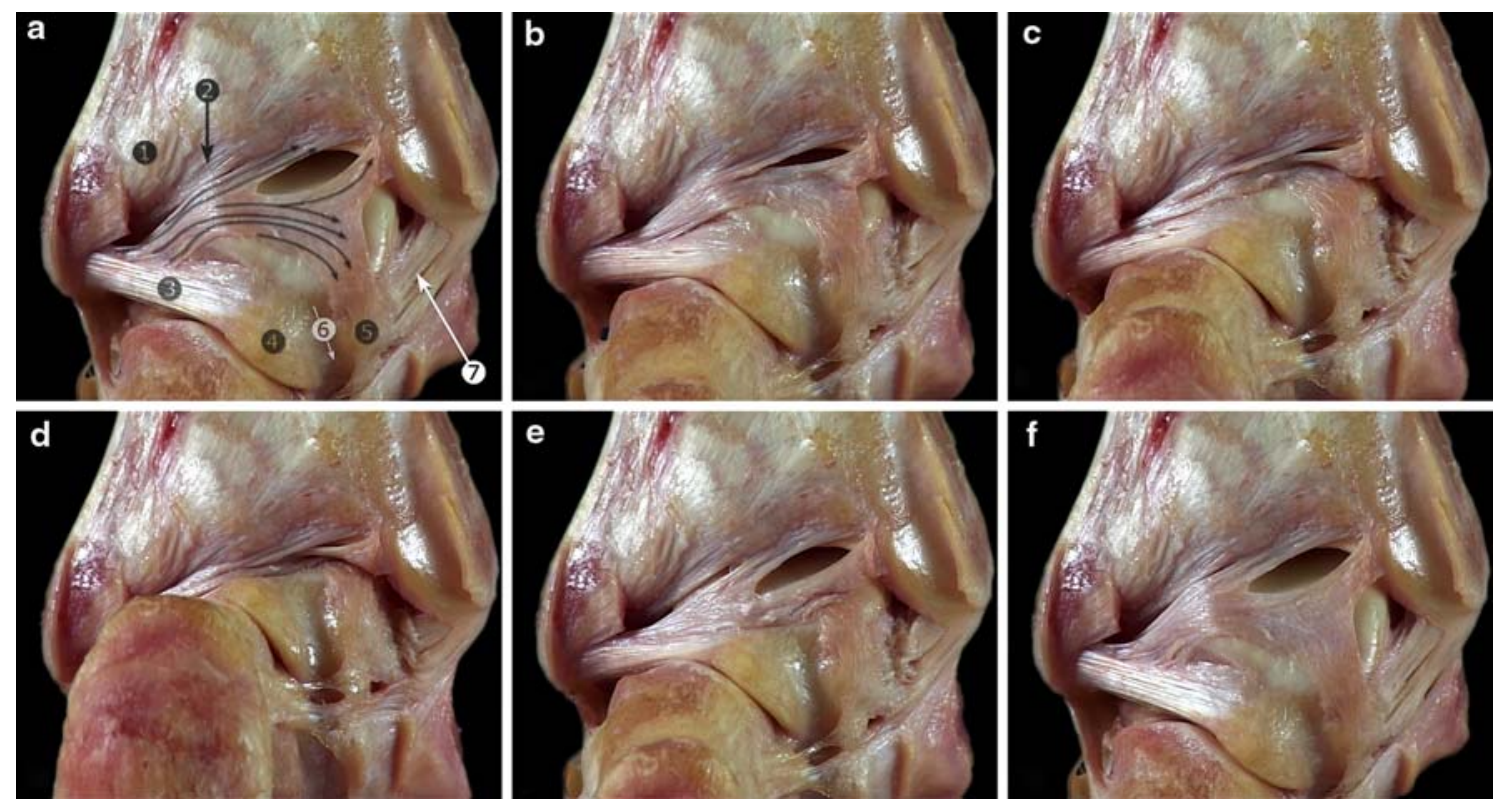

Fig. 11 Anatomic view of the posterior intermalleolar ligament (arrows) showing its involvement in the posterior soft tissue impingement of the ankle. From dorsiflexion (a) to plantar flexion (d), to dorsiflexion (f). 1 Superficial component of the posterior tibiofibular ligament; 2 deep component of the posterior tibiofibular ligament or transverse ligament; 3 posterior talofibular ligament; 4 lateral talar process; 5 medial talar process; 6 tunnel for the flexor hallucis longus tendon; 7 deep layer of the medial collateral ligament (deep posterior tibiotalar ligament)
Table 1 Comparison of the nomenclature used for the medial collateral components, as suggested by Sarrafian [36] and Milner and Soames [24]

\begin{tabular}{ll}
\hline Milner and Soames [24] & Sarrafian [36] \\
\hline Superficial layer & \\
Tibiospring ligament (major component) & $\begin{array}{l}\text { Tibioligamentous fascicle } \\
\text { Tibionavicular ligament (major component) } \\
\text { superficial tibiotalar fascicle }\end{array}$ \\
Superficial tibiotalar ligament (additional band) & Superficial posterior tibiotalar ligament \\
Tibiocalcaneal ligament (additional band) & Tibiocalcaneal ligament \\
Deep layer & \\
Deep posterior tibiotalar ligament (major component) & Deep posterior tibiotalar ligament \\
Anterior deep tibiotalar ligament (additional band) & Deep anterior tibiotalar ligament \\
\hline
\end{tabular}

interosseous tibiofibular ligament. The inferior segment of the interosseous membrane also helps stabilize the tibiofibular syndesmosis. Distal to the insertion site of this ligament, the remaining anterior surface corresponds to the tibiofibular synovial recess of the ankle joint, and at the posterior surface, there is a small bundle of adipose tissue called the fatty synovial fringe (Fig. 15). The synovial fringe lowers or rises during ankle movements, retracting in dorsiflexion to rise and position itself between the tibia and fibula and descending in plantar flexion toward the ankle joint. This structure has been implicated as a cause of chronic pain following ankle sprain in the condition known as anterolateral soft tissue impingement or more specifically, syndesmotic impingement.
Anterior or anteroinferior tibiofibular ligament

The ligament originates in the anterior tubercle of the tibia (5 $\mathrm{mm}$ in average above the articular surface [41]), and its fibers extend in a distal and lateral direction to the insertion site in the anterior margin of the lateral malleolus, with increased length of the fibers distally. Upon examination, the ligament is seen to be divided into several fascicles, allowing the perforation branches from the peroneal artery (Figs. 16, 17). The most distal fibers of the ligament at its origin may be confused with those of the anterior talofibular ligament $[1,6,36]$.

The most distal fascicle of the anterior tibiofibular ligament appears to be independent from the rest of the 


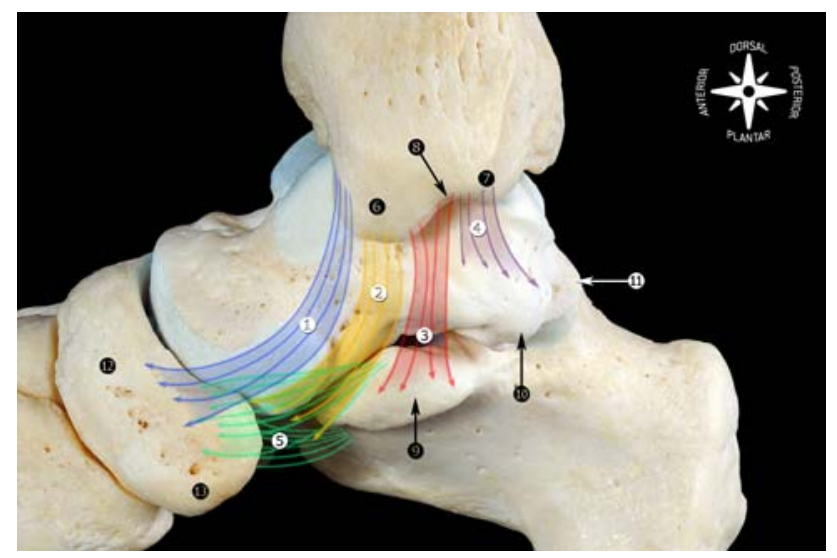

Fig. 12 Schematic representation of the main components of the medial collateral ligament found as frequently observed in our dissections. The morphology of the medial malleolus is helpful to understand the origins of the medial collateral ligament. In the medial view, two areas or segments (culliculi) can be seen, separated by the intercollicular groove. 1 Tibionavicular ligament; 2 tibiospring ligament; 3 tibiocalcaneal ligament; 4 deep posterior tibiotalar ligament; 5 spring ligament complex (plantar and superomedial calcaneonavicular ligaments); 6 anterior culliculus; 7 posterior culliculus; 8 intercullicular groove; 9 sustentaculum tali; 10 medial talar process; 11 lateral talar process; 12 navicular; 13 navicular tuberosity

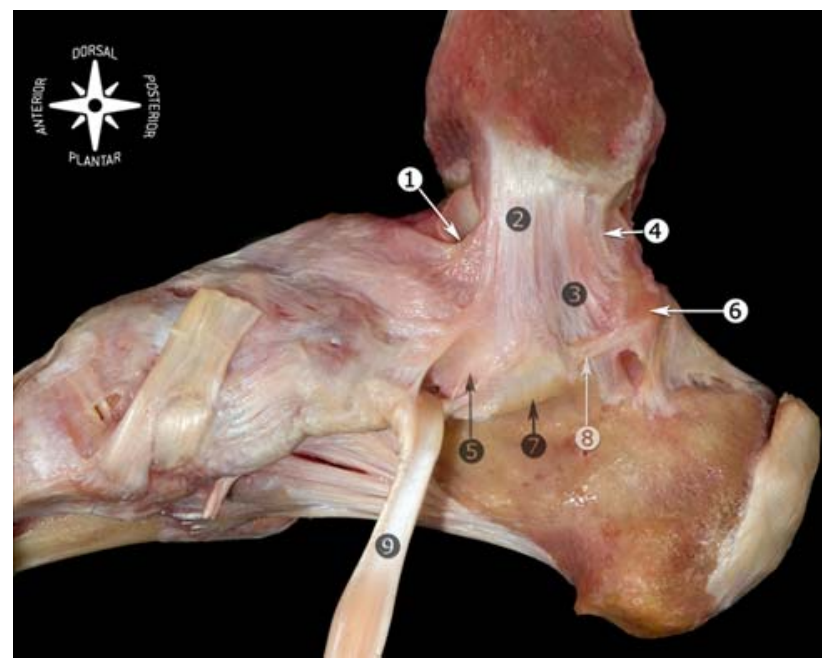

Fig. 13 Medial view of the anatomic dissection of the main components of the medial collateral ligament. 1 Tibionavicular ligament; 2 tibiospring ligament; 3 tibiocalcaneal ligament; 4 deep posterior tibiotalar ligament; 5 spring ligament complex (superomedial calcaneonavicular ligament); 6 medial talar process; 7 sustentaculum tali; 8 medial talocalcaneal ligament; 9 tibialis posterior tendon

structure (Fig. 16). It is separated by a septum of fibroadipose tissue and may be slightly deeper than the rest of the ligament. Pathology to this fascicle is frequently described as being responsible for anterolateral soft tissue impingement [1, 2, 5, 32]. Excision of this distal fascicle through open or ankle arthroscopic approach frequently resolves the patient's complaints, whereas the ankle stability is not comprised $[6,25,26,31]$.

Impingement of the distal fascicle of the anterior tibiofibular ligament appears to depend on changes in the ankle mechanics [6]. An injury to the LCL (e.g., anterior talofibular ligament) would increase anteroposterior laxity of the ankle [20]. This, in turn, would result in increased anterior extrusion of the talus and cause the distal fascicle to have greater contact and pressure on the talus [6]. Another factor related to distal fascicle impingement is the level at which the anterior tibiofibular ligament is inserted in the fibula with respect to the joint line. More distal insertion of the ligament could lead to increased contact in the neutral position of the ankle and a higher potential for ligamentous pathology (Fig. 18). Knowledge of this configuration is important to understand the anatomic bases for anterolateral soft tissue impingement, since abrasion between the distal fascicle of the anterior tibiofibular ligament and the talus may lead to pain (Fig. 19).

Our observations in the dissection room have allowed us to identify contact between the distal fascicle and the talus in the neutral position. This finding is frequently observed during ankle arthroscopy, and the surgeon should consider it a normal feature [2]. This fact has been reported by other authors [1, 19, 22, 26, 32], although in cases of anatomic variation or ankle instability, the feature may be pathological. Contact decreases with joint distraction [2], which should be taken into account during arthroscopy. Akseki et al. [2] observed that section of the anterior talofibular ligament does not alter the contact when the ankle is in neutral position, although important changes are observed when the ankle is in movement. Therefore, ankle instability is one direct factor in anterior tibiofibular ligament pathology.

A diagnosis of this type of ligamentous impingement should be considered in patients with chronic pain in the anterolateral area of the ankle following a sprain, with joint stability and a normal radiological appearance [14].

\section{Posterior or posteroinferior tibiofibular ligament}

As is frequently observed, also for this rather strong compact syndesmotic ligament, numerous terminologies have been postulated [5], which is particularly evident in the arthroscopic literature [16].

This ligament is basically formed by two independent components, the superficial and deep component (Fig. 20). 

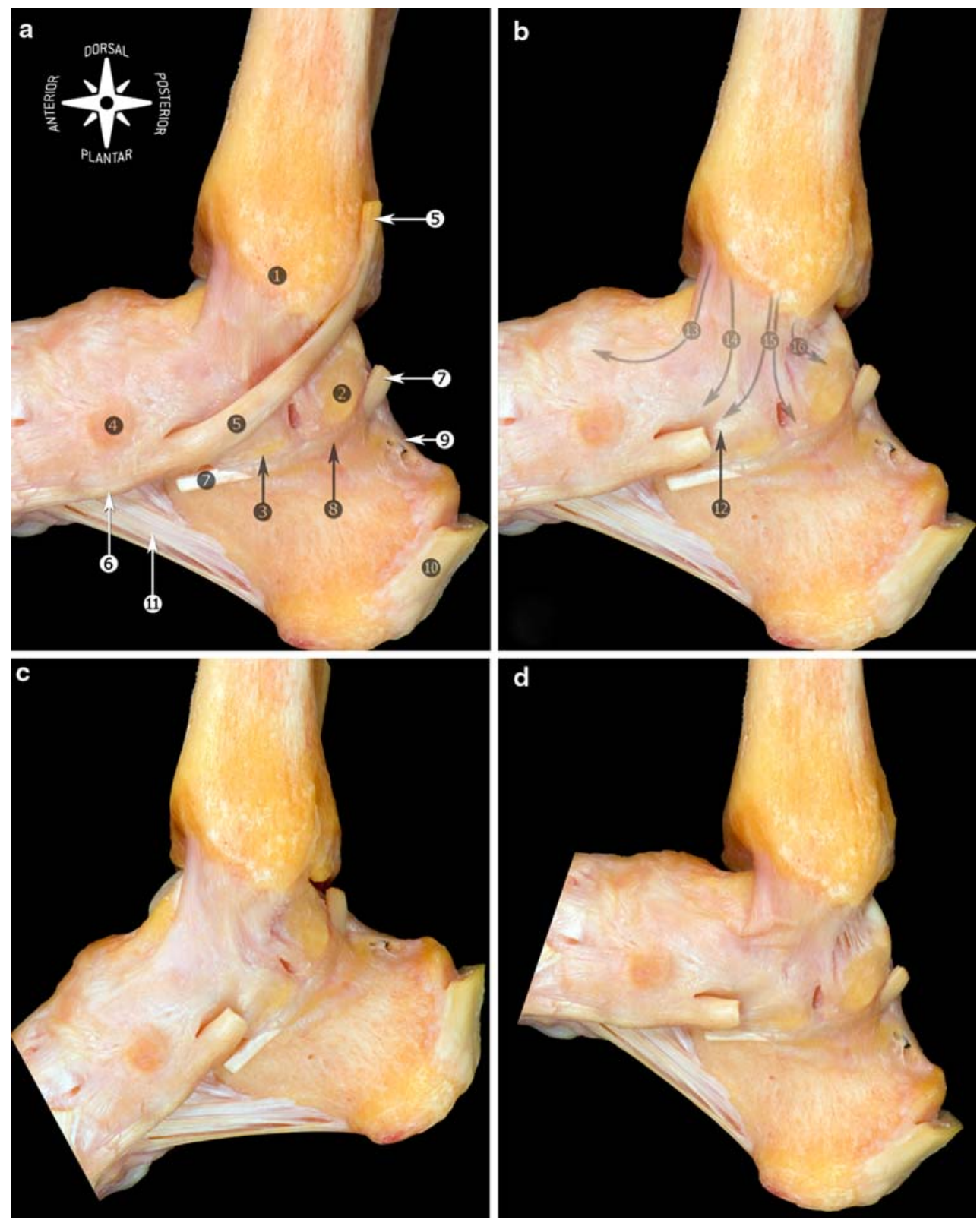

Fig. 14 Medial view of the anatomic dissection of the medial collateral ligament. Most of the medial collateral ligament is covered by tendons (tibialis posterior and flexor digitorum longus tendons). In order to see the ligament, the tendon of flexor digitorum longus was removed. a Neutral position showing the relationship with the tibialis posterior tendon. b The posterior tibialis tendon was removed. c Plantar flexion. The components located anteriorly to the bimalleolar axis are tensed. d Dorsiflexion. The components located anteriorly to

The superficial component originates at the posterior edge of the lateral malleolus and directs proximally and medially to insert in the posterior tibial tubercle. This component would be homologous to the anterior tibiofibular ligament. The term posterior or posteroinferior tibiofibular ligament is usually used to refer to the superficial component (Figs. 20, 21).

the bimalleolar axis are relaxed. 1 Medial malleolus; 2 lateral talar process; 3 sustentaculum tali; 4 navicular; 5 tibialis posterior tendon; 6 navicular tuberosity; 7 flexor hallucis longus (cut); 8 flexor hallucis longus retinaculum; 9 posterior talocalcaneal ligament; 10 calcaneal tendon (cut at the level of the insertion); 11 long plantar ligament; 12 spring ligament complex (superomedial calcaneonavicular ligament); 13 tibionavicular ligament; 14 tibiospring ligament; 15 tibiocalcaneal ligament; 16 deep posterior tibiotalar ligament

The deep component is cone shaped and originates in the proximal area of the malleolar fossa to insert in the posterior edge of the tibia. Its insertion is immediately posterior to the cartilaginous covering of the inferior tibial articular surface; the fibers may reach the medial malleolus (Fig. 21). This component is also known as the transverse ligament, forming a true labrum [36] to provide 


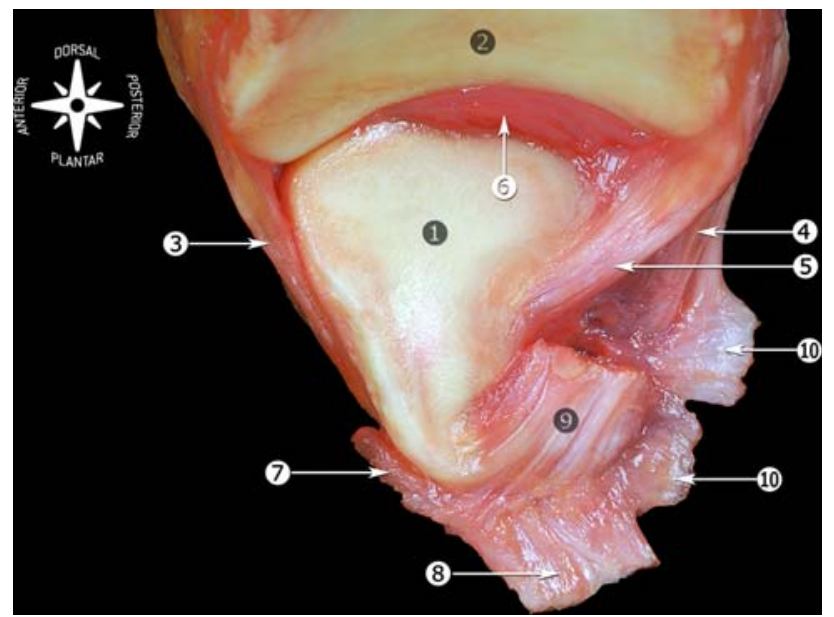

Fig. 15 Medial view of the tibiofibular joint (os talus previously removed). 1 Articular surface of the lateral malleolus; 2 distal articular surface of the tibia; 3 anterior tibiofibular ligament (distal fascicle); 4 superficial component of the posterior tibiofibular ligament; 5 deep component of the posterior tibiofibular ligament or transverse ligament; 6 fatty synovial fringe; 7 anterior talofibular ligament; 8 calcaneofibular ligament; 9 posterior talofibular ligament; 10 fibulotalocalcaneal ligament or Rouvière and Canela ligament

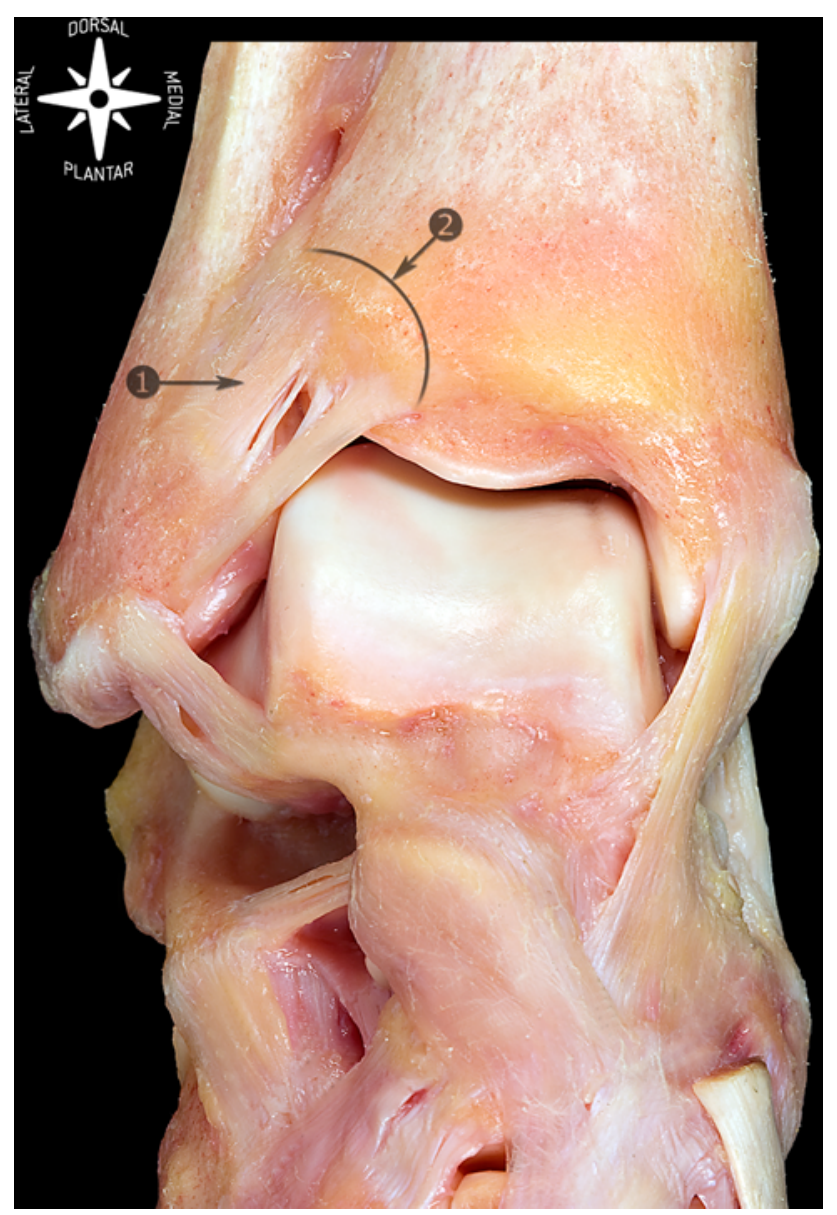

Fig. 16 Anterosuperior view of talocrural joint and dorsum of the foot. 1 Anterior tibiofibular ligament; 2 anterior tubercle of the tibia

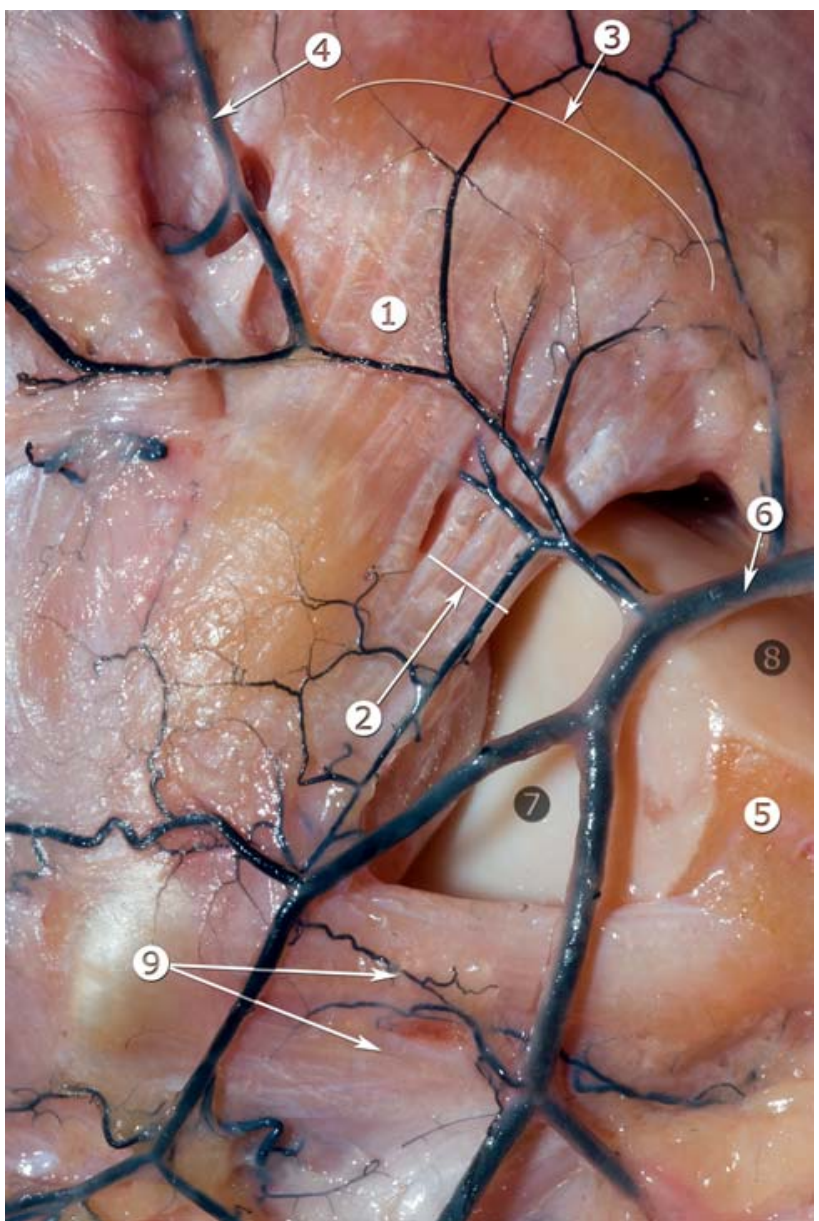

Fig. 17 Anatomic view of the anterolateral part of the ankle showing the relationship between the anterior tibiofibular ligament and the perforating branch of the peroneal artery (arteries are filled with black latex). 1 Anterior tibiofibular ligament; 2 distal fascicle of the anterior tibiofibular ligament; 3 anterior tubercle of the tibia; 4 perforating branch of peroneal artery; 5 triangular region of the talus; 6 anterior malleolar artery; 7 lateral articular surface of the talus; 8 dorsal articular surface of the talus; 9 anterior talofibular ligament

talocrural joint stability and to prevent posterior talar translation [37].

\section{Interosseous tibiofibular ligament}

The interosseous tibiofibular ligament is a dense mass of short fibers, which, together with adipose tissue and small branching vessels from the peroneal artery, span the tibia to the fibula. It can be considered a distal continuation of the interosseous membrane at the level of the tibiofibular syndesmosis [18, 36, 38]. Some investigators have suggested that the interosseous ligament is mechanically insignificant, whereas others consider it the primary bond between the tibia and fibula. Hoefnagels et al. [18] suggest that the interosseous ligament plays an important role in the stability of the ankle. 


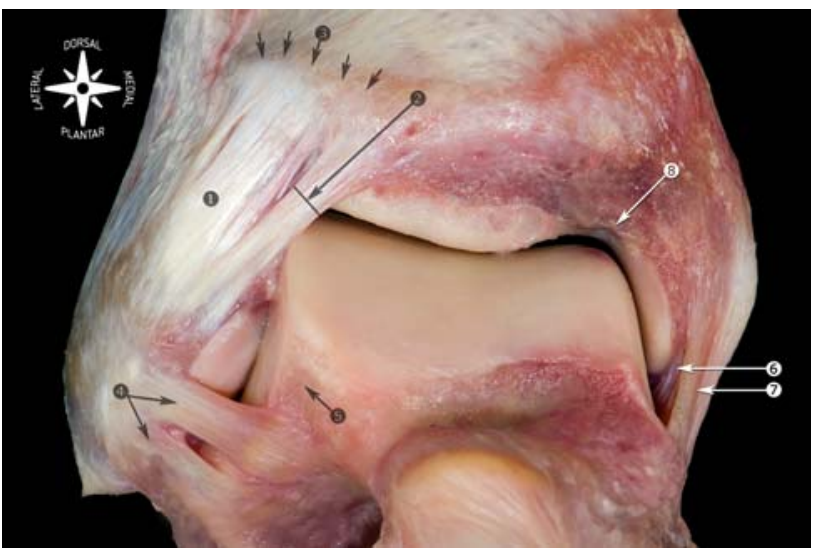

Fig. 18 Anatomic view of the anterior ligaments of the ankle. 1 Anterior tibiofibular ligament; 2 distal fascicle of the anterior tibiofibular ligament; 3 tibia (anterior tubercle indicated with arrows); 4 anterior talofibular ligament; 5 beveled triangular region of the talus; 6 deep layer of the medial collateral ligament; 7 superficial layer of the medial collateral ligament; 8 notch of Harty

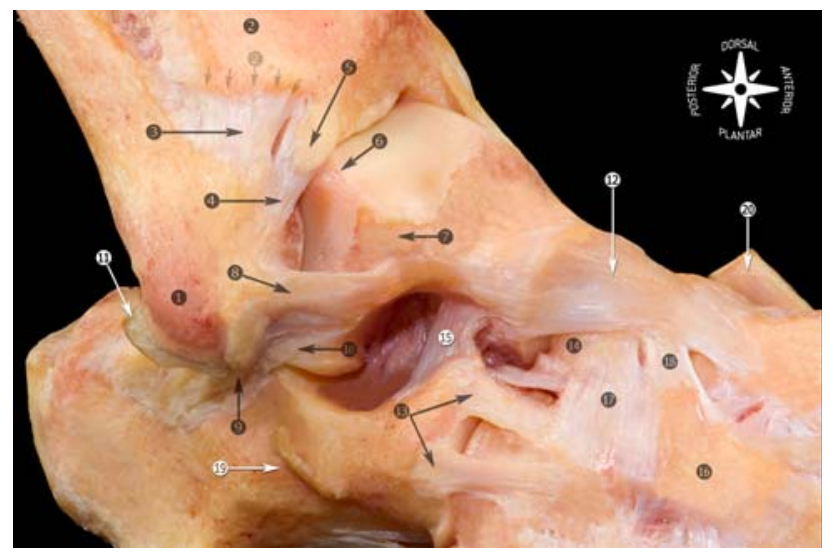

Fig. 19 Osteoarticular anatomic dissection of the ligaments of the foot and ankle joint. 1 Tip of the lateral malleolus; 2 tibia (anterior tubercle indicated with arrows); 3 anterior tibiofibular ligament; 4 distal fascicle of the anterior tibiofibular ligament; 5 imaging showing a calcification in the tibial insertion of the distal fascicle of the anterior tibiofibular ligament; 6 abrasion of the joint cartilage in the region where the anterior tibiofibular ligament came into contact with the talus; 7 beveled triangular region of the talus; 8 anterior talofibular ligament; 9 calcaneofibular ligament; 10 lateral talocalcaneal ligament; 11 cartilaginous rim; 12 talonavicular ligament; 13 lateral calcaneocuboid ligament; 14 navicular; 15 cervical ligament; 16 lateral cuneiform; 17 dorsal cuboideonavicular ligament; 18 dorsal cuneonavicular ligament; 19 calcaneus (peroneal tubercle); 20 anterior tibialis tendon

\section{Conclusions}

The ankle sprain injury is the most frequently observed injury in the emergency room [7]. Up to $40 \%$ of individuals with a history of an ankle ligament injury have been found to have residual complaints interfering with daily living $[15,42]$. Adequate knowledge of the anatomy of the ankle

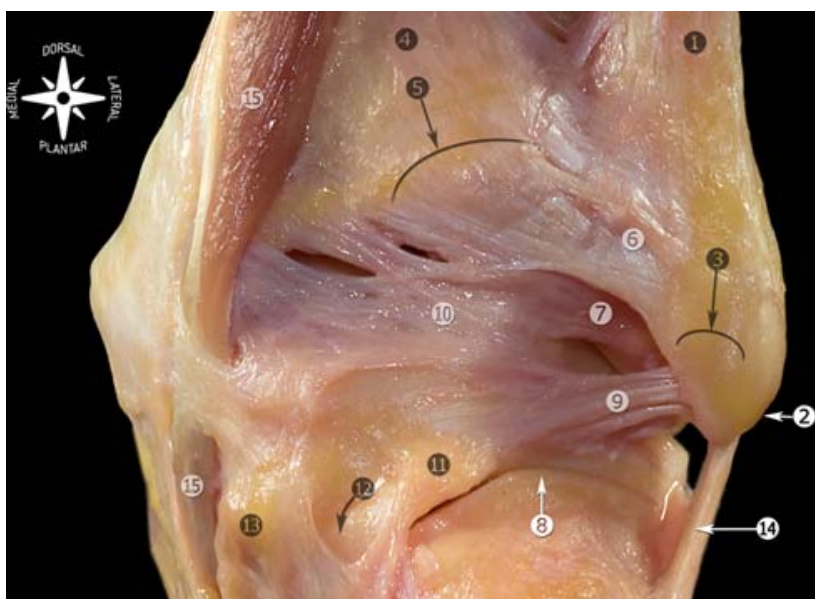

Fig. 20 Anatomic dissection of the posterior ligaments of the ankle. 1 Lateral malleolus; 2 tip of the lateral malleolus; 3 peroneal groove; 4 tibia; 5 posterior tubercle of the tibia; 6 posterior tibiofibular ligament, superficial component; 7 posterior tibiofibular ligament, deep component or transverse ligament; 8 subtalar joint; 9 posterior talofibular ligament; 10 posterior intermalleolar ligament; 11 lateral talar process; 12 tunnel for flexor hallucis longus tendon (tendon was removed); 13 medial talar process; 14 calcaneofibular ligament; 15 flexor digitorum longus

ligaments provides a foundation for understanding the basic mechanism of injury, diagnosis, and treatment of these ankle sprains.

Soft tissue impingement syndromes of the ankle are usually preceded by an ankle sprain. Depending on the mechanism of injury, a specific ligament and/or ligaments can be injured [43]. Injury to the anterior talofibular ligament is the most common injury following an ankle sprain. Most frequently, it is an isolated injury; however, in approximately $20 \%$ of the patients, also the calcaneofibular ligament is injured [9].

An inversion sprain can result in injury to the capsule, lateral or medial collateral ligaments, or tibiofibular ligaments. The added influence of plantar or dorsiflexion on the injury mechanism will mean that the lesion is predominantly anterior or posterior, respectively, and could also lead to injury to other structures such as the posterior intermalleolar ligament, the osteochondral region of the neck of the talus, or the anteroinferior margin of the tibia.

The mechanism of foot eversion is more closely associated with injury to the medial capsular and ligamentous elements, although an inversion sprain can also produce a lesion to these structures. Medial injury is probably more influenced by the rotating component of the subtalar joint to which the capsule and the MCL are subject.

The aim of this pictorial review on the anatomy of the ankle ligaments is to provide a guide to those who are involved in diagnosing and treating ligament injury around the ankle. 


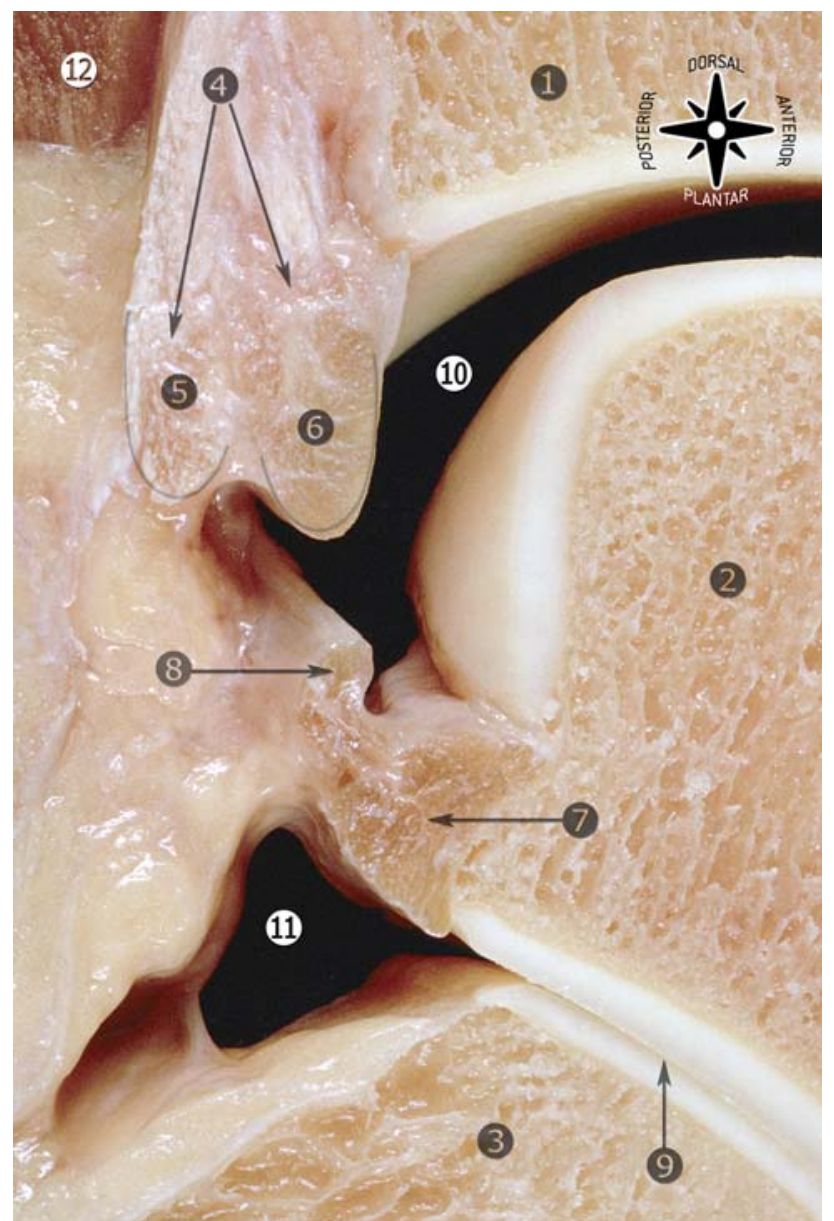

Fig. 21 Sagittal section of the ankle (lateral view). 1 Tibia; 2 talus; 3 calcaneus; 4 posterior tibiofibular ligament; 5 superficial component of the posterior tibiofibular ligament; 6 deep component of the posterior tibiofibular ligament or transverse ligament; 7 posterior talofibular ligament; 8 posterior intermalleolar ligament; 9 subtalar joint; 10 talocrural joint; 11 posterior capsular recess of the subtalar joint; 12 flexor hallucis longus muscle

Acknowledgments No benefits in any form have been received or will be received from a commercial party related directly or indirectly to the subject of this review. No sources of funding were received to assist in this review. The authors have no conflicts of interest that are directly relevant to this review.

Open Access This article is distributed under the terms of the Creative Commons Attribution Noncommercial License which permits any noncommercial use, distribution, and reproduction in any medium, provided the original author(s) and source are credited.

\section{References}

1. Akseki D, Pinar H, Bozkurt M et al (1999) The distal fascicle of the anterior inferior tibiofibular ligament as a cause of anterolateral ankle impingement. Results of arthroscopic resection. Acta Orthop Scand 70:478-482

2. Akseki D, Pinar H, Yaldiz K et al (2002) The anterior inferior tibiofibular ligament and talar impingement: a cadaveric study. Knee Surg Sports Traumatol Arthrosc 10:321-326
3. Anderson KJ, Lecoq JF (1954) Operative treatment of injury to the fibular collateral ligament of the ankle. J Bone Joint Surg Am 36:825-832

4. Balduini FC, Tetzlaff J (1982) Historical perspectives on injuries of the ligaments of the ankle. Clin Sports Med 1:3-12

5. Bartonícek J (2003) Anatomy of the tibiofibular syndesmosis and its clinical relevance. Surg Radiol Anat 25:379-386

6. Bassett FH III, Gates HS III, Billys JB et al (1990) Talar impingement by the anteroinferior tibiofibular ligament. A cause of chronic pain in the ankle after inversion sprain. J Bone Joint Surg Am 72:55-59

7. Boruta PM, Bishop JO, Braly WG et al (1990) Acute ankle ligament injuries; a literature review. Foot Ankle 11:107-113

8. Boss AP, Hintermann B (2002) Anatomical study of the medial ankle ligament complex. Foot Ankle Int 23:547-553

9. Broström L (1966) Sprained ankles V. Treatment and prognosis in recent ligament ruptures. Acta Chir Scand 132:537-550

10. Burks RT, Morgan J (1994) Anatomy of the lateral ankle ligaments. Am J Sport Med 22:72-77

11. Clanton TO, Paul P (2002) Syndesmosis injuries in athletes. Foot Ankle Clin N Am 7:529-549

12. Delfaut EM, Demondion X, Boutry N et al (2003) Multi-fasciculated anterior talo-fibular ligament: reassessment of normal findings. Eur Radiol 13:1836-1842

13. Ebraheim NA, Taser F, Shafiq Q et al (2006) Anatomical evaluation and clinical importance of the tibiofibular syndesmosis ligaments. Surg Radiol Anat 28:142-149

14. Ferkel RD (1994) Differential diagnosis of the chronic ankle sprain pain in the athlete. Sports Med Arthroscopy Rev 2:274-283

15. Gerber JP, Williams GN, Scoville CR et al (1998) Persistent disability associated with ankle sprains: a prospective examination of an athletic population. Foot Ankle Int 19:653-660

16. Golanó P, Mariani PP, Rodríguez-Niedenfuhr M et al (2002) Arthroscopic anatomy of the posterior ankle ligaments. Arthroscopy $18: 353-358$

17. Hamilton WG, Gepper MJ, Thompson FM (1996) Pain in the posterior aspect of the ankle in dancers. Differential diagnosis and operative treatment. J Bone Joint Surg Am 78:1491-1500

18. Hoefnagels EM, Waites MD, Wing ID et al (2007) Biomechanical comparison of the interosseous tibiofibular ligament and the anterior tibiofibular ligament. Foot Ankle Int 28:602-604

19. Horner G, Liu S (1996) Arthroscopic treatment of talar impingement by the accessory anteroinferior tibiofibular ligament. Arthroscopy 12:384

20. Johnson EE, Markolf KL (1983) The contribution of the anterior talofibular ligament to ankle laxity. J Bone Joint Surg Am 65:81-88

21. Kapanji IA (1982) Cuadernos de fisiología articular (Joint physiology). Cuaderno III. Barcelona:Masson, SA, pp 174-177

22. Liu SH, Raskin A, Osti L et al (1994) Arthroscopic treatment of anterolateral ankle impingement. Arthroscopy 10:215-218

23. Milner CE, Soames RW (1997) Anatomical variations of the anterior talofibular ligament of the human ankle joint. J Anat 191:457-458

24. Milner CE, Soames RW (1998) Anatomy of the collateral ligaments of the human ankle joint. Foot Ankle Int 19:757-760

25. Nikolopoulos CE (1982) Anterolateral instability of the ankle joint. An anatomical, experimental and clinical study. Thesis, University of Athenes, Athenes, Greece

26. Nikolopoulus CE, Tsirikos AI, Sourmelis S et al (2004) The accessory anteroinferior tibiofibular ligament as a cause of talar impingement. A cadaveric study. J Sports Med 32:389-395

27. Oh CS, Won HS, Chung IH et al (2006) Anatomic variations and MRI of intermalleolar ligament. AJR 186:943-947

28. Pankovich AM, Shivaram MS (1979) Anatomical basis of variability in injuries of the medial malleolus and the deltoid ligament. I. Anatomical studies. Acta Orthop Scand 50:217-223 
29. Paturet G (1951) Traité d'anatomie humaine (A treatise on human anatomy). París:Masson, pp 704-711

30. Peace KAL, Hillier JC, Hulme A et al (2004) MRI features of posterior ankle impingement syndrome in ballet dances: a review of 25 cases. Clin Radiol 59:1025-1033

31. Rasmussen O, Tovborg-Jensen I, Boe S (1982) Distal tibiofibular ligaments, analysis of function. Acta Orthop Scand 53:681686

32. Ray RG, Kriz BM (1991) Anterior inferior tibiofibular ligament. Variations and relationship to the talus. J Am Podiatr Med Assoc $81: 479-485$

33. Renstrom FH, Lynch SA (1999) Acute injuries of the ankle. Foot Ankle Clin 4:697-711

34. Rosenberg ZS, Cheung YY, Beltran J et al (1995) Posterior intermalleolar ligament of the ankle: normal anatomy and MR imaging features. AJR 165:387-390

35. Ruth CJ (1961) The surgical treatment of injuries of the fibular collateral ligaments of the ankle. J Bone Joint Surg Am 43:233236

36. Sarrafian SK (1993) Anatomy of the foot and ankle. Descriptive, topographic, functional, 2nd edn. Lippincott, Philadelphia, pp 159-217

37. Taylor DC, Englehardt DL, Bassett FH (1992) Syndesmosis sprains of the ankle. The influence of heterotopic ossification. Am J Sport Med 20:146-150
38. Testut L, Latarjet A (1985) Tratado de anatomía humana (A treatise on human anatomy). Barcelona:Salvat Editores, SA, pp 704-719

39. Trouilloud P, Dia A, Grammont P et al (1988) Variations du ligament calcaneo-fibulaire. Aplications à la cinématique de la cheville (Variations in the calcaneofibular ligament. Applications to ankle kinetics). Bull Assoc Anat 72:31-35

40. van den Bekerom MPJ, Oostra RJ, Golanó P et al (2008) The anatomy in relation to injury of the lateral collateral ligaments of the ankle: a current concepts review. Clin Anat 21:619-626

41. van den Bekerom MPJ, Raven EEJ (2007) The distal fascicle of the anterior inferior tibiofibular ligament as a cause of tibiotalar impingement syndrome: a current concepts review. Knee Surg Sports Traumatol Arthosc 15:465-471

42. Verhagen RA, de Keizer G, van Dijk CN (1995) Long-term follow-up of inversion trauma of the ankle. Arch Orthop Trauma Surg 114:92-96

43. van Dijk CN (1994) On diagnostic strategies in patient with severe ankle sprain. Thesis, University of Amsterdam, The Netherlands

44. van Dijk CN, Scholten PE, Krips R (2000) A 2-portal endoscopic approach for diagnosis and treatment of posterior ankle pathology. Technical note. Arthroscopy 16:871-876

45. van Dijk CN, de Leeuw PA, Scholten PE (2009) Hindfoot endoscopy for posterior ankle impingement. Surgical technique. J Bone Joint Surg Am 2:287-298 